\title{
Some Invariants for Conics and Their Applications
}

\author{
By \\ Isao NARUKI*
}

1. Compared with the case of lines, very little is known about the question: what kind of configurations of conics are possible in the (complex) projective plane. In this note we attempt to introduce some analytic invariants to control the intersection behavior of conics; if one wants to study the configurations of conics with a prescribed contact behavior, these invariants might serve as the natural parameters which they depend on. In fact we will describe the parameter space (the moduli) for some elementary configurations which may often appear as parts of some more complicated ones. We will also clarify how the invariants behave under duality. As a by-product we obtain three families of quartic surfaces in $\boldsymbol{P}_{3}$ with three singular points of type $A_{3}$ and seven ordinary double points $\left(A_{1}\right)$ : two of them are dual to each other and the remaining one is self-dual.

This work was originally motivated by the problem of finding interesting abelian covers of $\boldsymbol{P}_{\mathbf{2}}$ branched over several conics. The author would like to express his sincere thanks to Professor Hirzebruch for the introduction to this problem. He is also very grateful to Professor Brieskorn for the discussion which led to the generalization of the invariants.

2. All conics in this note are assumed to be non-degenerate. A finite union of distinct conics in the projective plane is called a configuration; in any configuration discussed in this note, no three conics are allowed to meet at one point, unless the contrary is explicitly stated; namely, we exclude any kind of triple intersection points. We also exclude contacts of order higher than 2 from our configurations. We have thus only ordinary intersection points and tacnodes.

3. Suppose that we are given two conics $Q_{1}: q_{1}(x, y, z)=0, Q_{2}: q_{2}(x, y, z)$

Received May 19, 1983.

* Research Institute for Mathematical Sciences, Kyoto University, Kyoto 606, Japan. Partially supported by the SFB $40 》 \mid$ Theoretische Mathematik《 and the Max-PlanckInstitut für Mathematik in Bonn. 
$=0$ in the complex projective plane $\boldsymbol{P}_{2}:(x, y, z)$ and that they are in the general position (i.e. they intersect in four points). Denote further the intersection points by $p_{0}, p_{1}, p_{2}, p_{3}$ and the $(2,2)$ - partitions $\left\{p_{0}, p_{1} ; p_{2}, p_{3}\right\},\left\{p_{0}, p_{2} ; p_{1}, p_{3}\right\}$, $\left\{p_{0}, p_{3} ; p_{1}, p_{2}\right\}$ by $\iota_{1}, \iota_{2}, \iota_{3}$. The partitions are called the references of the pair $\left\{Q_{1}, Q_{2}\right\}$. They are in a natural one to one correspondence with the singular members of the pencil $\mathscr{P}=\{Q(t)\}$ generated by $Q_{1}, Q_{2}$ :

$$
Q(t): t q_{1}+q_{2}=0 .
$$

(Note that $Q(\infty)=Q_{1}, Q(0)=Q_{2}$ with this notation.) The singular members correspond to the roots $t_{1}, t_{2}, t_{3}$ of the cubic equation

$$
\operatorname{det}\left(t q_{1}+q_{2}\right)=0
$$

where the determinant is taken by regarding quadratic forms as symmetric matrices in the usual way. By changing the indices, we can assume that $Q\left(t_{1}\right)$ $=\overline{p_{0} p_{1}} \cup \overline{p_{2} p_{3}}, Q\left(t_{2}\right)=\overline{p_{0} p_{2}} \cup \overline{p_{1} p_{3}}, Q\left(t_{3}\right)=\overline{p_{0} p_{3}} \cup \overline{p_{1} p_{2}}$ where $\overline{p_{i} p_{j}}$ denotes the line passing through $p_{i}, p_{j}$ for each $(i, j)$. Thus $\iota_{1}, \iota_{2}, \iota_{3}$ correspond to $Q\left(t_{1}\right)$, $Q\left(t_{2}\right), Q\left(t_{3}\right)$. Our first invariant is now defined for ordered pairs of two conics and their references; we set

$$
\left\{\begin{array}{l}
{\left[Q_{2} / Q_{1} ; \iota_{1}\right]=t_{1}^{2} /\left(t_{2} t_{3}\right)} \\
{\left[Q_{2} / Q_{1} ; \iota_{2}\right]=t_{2}^{2} /\left(t_{1} t_{3}\right)} \\
{\left[Q_{2} / Q_{1} ; \iota_{3}\right]=t_{3}^{2} /\left(t_{1} t_{2}\right)}
\end{array}\right.
$$

We have some obvious properties:

$$
\left\{\begin{array}{l}
{\left[Q_{2} / Q_{1} ; \iota_{i}\right] \cdot\left[Q_{1} / Q_{2} ; \iota_{i}\right]=1 \quad i=1,2,3} \\
{\left[Q_{2} / Q_{1} ; \iota_{1}\right] \cdot\left[Q_{2} / Q_{1} ; \iota_{2}\right] \cdot\left[Q_{2} / Q_{1} ; \iota_{3}\right]=1}
\end{array}\right.
$$

Projective invariance of these quantities follows from the fact that the coordinate $t$ of the pencil $\mathscr{P}$ such that $t=0, \infty$ represents $Q_{2}, Q_{1}$ is unique up to a multiplicative constant. But it can also be proved in the following way. We choose the (unique) coordinate $\tau$ of $\mathscr{P}$ such that $\tau=1,0, \infty$ correspond to $Q\left(t_{1}\right), Q\left(t_{2}\right)$, $Q\left(t_{3}\right)$; explicitly $\tau=\left(t_{1}-t_{3}\right) \cdot\left(t-t_{2}\right) /\left(\left(t_{1}-t_{2}\right) \cdot\left(t-t_{3}\right)\right)$. Suppose that $\tau=\alpha, \beta$ correspond to $Q_{1}, Q_{2}$. Then we have

$$
\left[Q_{2} / Q_{1} ; \iota_{1}\right]=\alpha \cdot(\beta-1)^{2} /\left(\beta \cdot(\alpha-1)^{2}\right) .
$$

4. Now we discuss some degenerate cases in which the invariants introduced above are still well-defined and are expressed by a unified invariant. We say that two conics are in a special position if they are tangent to each other at least 
at one point; more specifically, they are said to be in (2)-contact (resp. (2, 2)contact) if they are tangent at exactly one point (resp. at two points). According to the assumption in Section 2 we have excluded contacts of order 3, 4. Now suppose the conics $Q_{1}: q_{1}=0, Q_{2}: q_{2}=0$ are in special position. Then the equation

$$
\operatorname{det}\left(t q_{1}+q_{2}\right)=0
$$

has one simple root $t^{\prime}$ and one double root $t^{\prime \prime}$. The singular member $Q\left(t^{\prime}\right)$ contains the common tangent(s) while $Q\left(t^{\prime \prime}\right)$ contains the contact point(s) in its singular locus. We have also only two references $\iota^{\prime}, \iota^{\prime \prime}$ of the pair $\left\{Q_{1}, Q_{2}\right\}$ corresponding to $t^{\prime}, t^{\prime \prime} ; c^{\prime}$ is the single one and $\iota^{\prime \prime}$ the double one. We now set for two conics in a special position:

$$
\left[Q_{2} / Q_{1}\right]=t^{\prime} / t^{\prime \prime}
$$

The projective invariance of this quantity is obvious and we have the identity:

$$
\left\{\begin{array}{l}
{\left[Q_{2} / Q_{1} ; \iota^{\prime}\right]=\left[Q_{2} / Q_{1}\right]^{2}} \\
{\left[Q_{2} / Q_{1} ; \iota^{\prime \prime}\right]=\left[Q_{2} / Q_{1}\right]^{-1}=\left[Q_{1} / Q_{2}\right] .}
\end{array}\right.
$$

In view of the last identity the invariant $\left[Q_{2} / Q_{1}\right]$ should be regarded as being among the old ones.

5. In this section we want to show that our invariants enjoy some multiplicative properties. We begin with the fact that, given two conics, there are in general three families of conics which are in $(2,2)$-contact with both of them, and that they correspond to the references of the two conics. Recall, therefore, that in Section $3 p_{0}, p_{1}, p_{2}, p_{4}$ are the intersection points of $Q_{1}: q_{1}=0, Q_{2}: q_{2}=0$ and $Q\left(t_{1}\right), Q\left(t_{2}\right), Q\left(t_{3}\right)$ are the singular members of the pencil $\mathscr{P}=\left\{Q(t): t q_{1}\right.$ $\left.+q_{2}=0\right\}$; thus each quadratic form $q^{(i)}=t_{i} q_{1}+q_{2}$ factors into two linear forms $l^{(i)}, m^{(i)}$ :

$$
q^{(i)}=t_{i} q_{1}+q_{2}=l^{(i)} \cdot m^{(i)}, \quad i=1,2,3 .
$$

Now we can construct for each $i=1,2,3$ a one-parameter family $\mathscr{F}_{i}$ of conics

$$
\begin{aligned}
P^{(i)}(s): p^{(i)}(s) & =\left(s l^{(i)}+m^{(i)}\right)^{2}-4 s t_{i} q_{1} \\
& =\left(s l^{(i)}-m^{(i)}\right)^{2}+4 s q_{2}=0 .
\end{aligned}
$$

The first identity is the definition of $p^{(i)}(s)$. The second identity follows from (5.1). It is obvious that $P^{(i)}(s)$ is in $(2,2)$-contact with $Q_{1}, Q_{2}$; the tangent points of $P^{(i)}(s)$ with $Q_{1}$ (resp. $Q_{2}$ ) lie on the line $s l^{(i)}+m^{(i)}=0$ (resp. $s l^{(i)}-m^{(i)}=0$ ). 
Obviously these three families $\mathscr{F}_{i}$ correspond to the references $\iota_{i}(i=1,2,3)$ of $Q_{1}, Q_{2}$.

Proposition 5.1. Any conic which is in (2, 2)-contact with $Q_{1}, Q_{2}$ belongs to one of the three families $\mathscr{F}_{i}(i=1,2,3)$ above. If two families, say $\mathscr{F}_{2}$, $\mathscr{F}_{3}$, have a common (non-degenerate) member, then they coincide; that is, $t_{2}=t_{3}$. If $t_{2}=t_{3}$, then any member of $\mathscr{F}_{2}=\mathscr{F}_{3}$ is tangent to $Q_{1}$ and $Q_{2}$ at their contact point.

Note that the last case violates the assumptions of Section 2; namely, in case $Q_{1}, Q_{2}$ are in a special position, there is essentially one and only one family of conics which are in $(2,2)$-contact with $Q_{1}, Q_{2}$.

We turn to the general case: Given a conic $Q$ which is in $(2,2)$-contact with $Q_{1}, Q_{2}$, we can speak of the family to which it belongs and hence also of the reference to which this family corresponds. We can thus say that $Q$ belongs to the reference.

Proposition 5.2. Suppose that $Q, Q_{1}, Q_{2}$ are three conics such that $Q$ is in (2,2)-contact with $Q_{1}, Q_{2}$ and that c is the reference of $\left\{Q_{1}, Q_{2}\right\}$ to which $Q$ belongs. Then

$$
\left[Q_{2} / Q_{1} ; \iota\right]=\left[Q_{1} / Q\right] \cdot\left[Q / Q_{2}\right] .
$$

In particular, if $Q_{1}, Q_{2}$ are in a special position, then

$$
\left[Q_{2} / Q_{1}\right]^{2}=\left[Q_{1} / Q\right] \cdot\left[Q / Q_{2}\right] \text {. }
$$

6. Now we apply Proposition 5.2 to the problem of obtaining necessary conditions for three or four conics to form some interesting configurations. To begin with, we suppose that any two of the conics $Q_{1}, Q_{2}, Q_{3}$ are in $(2,2)$ contact with each other. Then, by Proposition 5.2, we have $\left[Q_{j} / Q_{i}\right]^{2}$ $=\left[Q_{i} / Q_{k}\right] \cdot\left[Q_{k} / Q_{j}\right]$ for any permutation $(i, j, k)$ of $(1,2,3)$. It follows that there is a third root of unity $\omega$ such that $\left[Q_{3} / Q_{2}\right]=\omega\left[Q_{1} / Q_{3}\right]=\omega^{2}\left[Q_{2} / Q_{1}\right]$. But one can show directly

Proposition 6.1. If $Q_{1}, Q_{2}, Q_{3}$ are pairwise in $(2,2)$-contact, then

$$
\left[Q_{3} / Q_{2}\right]=\left[Q_{1} / Q_{3}\right]=\left[Q_{2} / Q_{1}\right] \text {. }
$$

As a corollary of this we obtain

Proposition 6.1'. Suppose that four conics $Q_{1}, Q_{2}, Q_{3}, Q_{4}$ are pairwise in $(2,2)$-contact. Then we have $\left[Q_{i} / Q_{j}\right]=-1$ for $1 \leqq i \neq j \leqq 4$. 
Remark. Such four conics as in the proposition are projectively unique. They are given for example by

$$
\begin{aligned}
& Q_{1}: x^{2}+y^{2}+z^{2}=0 \\
& Q_{2}:-x^{2}+y^{2}+z^{2}=0 \\
& Q_{3}: x^{2}-y^{2}+z^{2}=0 \\
& Q_{4}: x^{2}+y^{2}-z^{2}=0 .
\end{aligned}
$$

Another direct consequence of Proposition 5.2 is the following:

Proposition 6.2. Suppose that $Q, Q_{1}, Q_{2}, Q_{3}$ are four conics such that $Q$ is in $(2,2)$-contact with all of $Q_{1}, Q_{2}, Q_{3}$. Let $\iota, \iota^{\prime}, \iota^{\prime \prime}$ be the references of $\left\{Q_{2}, Q_{3}\right\},\left\{Q_{1}, Q_{3}\right\},\left\{Q_{1}, Q_{2}\right\}$ to which $Q$ belongs. Then we have:

$$
\left[Q_{3} / Q_{2} ; \iota\right] \cdot\left[Q_{1} / Q_{3} ; \iota^{\prime}\right] \cdot\left[Q_{2} / Q_{1} ; \iota^{\prime \prime}\right]=1 \text {. }
$$

In particular, if $Q_{1}, Q_{2}, Q_{3}$ are pairwise in special position, then we have:

$$
\left[Q_{3} / Q_{2}\right] \cdot\left[Q_{1} / Q_{3}\right] \cdot\left[Q_{1} / Q_{2}\right]= \pm 1
$$

There must be a sharp geometric distinction between the cases where the + and - signs appear in (6.3). Moreover we should remark that (6.3) is not sufficient for the existence of $Q$ satisfying the condition of Proposition 6.2. To get more detailed conditions we need a new invariant, which we will introduce in the next section.

7. Suppose as above that $Q_{1}, Q_{2}, Q_{3}$ are three conics such that any two of them are tangent to each other at exactly one point. Let $p_{1}, p_{2}, p_{3}$ be the contact points of pairs $\left\{Q_{2}, Q_{3}\right\},\left\{Q_{1}, Q_{3}\right\},\left\{Q_{1}, Q_{2}\right\}$ and $l_{1}, l_{2}, l_{3}$ the common tangents at $p_{1}, p_{2}, p_{3}$. We denote further, for any permutation $(i, j, k)$ of $(1,2,3)$, the intersection point of $l_{j}$ and $l_{k}$ by $q_{i}$ and the intersection point of $l_{i}$ and the line $\overline{p_{j} p_{k}}$ by $\bar{p}_{i}$. We have thus four points $p_{i}, \bar{p}_{i}, q_{j}, q_{k}$ on each line $l_{i}$. One can now show that these three quadruplets of collinear points all have the same crossratio. With this in mind, we introduce the new invariant:

$$
\left[Q_{i}, Q_{j}, Q_{k}\right]=\left(q_{j}-p_{i}\right) \cdot\left(q_{k}-\bar{p}_{i}\right) /\left(\left(q_{j}-\bar{p}_{i}\right) \cdot\left(q_{k}-p_{i}\right)\right) \text {. }
$$

One sees immediately that this is invariant under the cyclic permutations of $Q_{i}, Q_{j}, Q_{k}$, so that we have only two quantities $\left[Q_{1}, Q_{2}, Q_{3}\right],\left[Q_{3}, Q_{2}, Q_{1}\right]$ which are each other's inverse. We also have the following non-trivial relation:

$$
\left[Q_{2} / Q_{1}\right] \cdot\left[Q_{3} / Q_{2}\right] \cdot\left[Q_{1} / Q_{3}\right]=-\left[Q_{1}, Q_{2}, Q_{3}\right]^{3} \text {. }
$$

The desired strengthening of Proposition 6.2 is now the following: 
Proposition 7.1. Suppose that three conics $Q_{1}, Q_{2}, Q_{3}$ are pairwise in special positions. Then there exists a conic $Q$ which is in $(2,2)$-contact with all of $Q_{1}, Q_{2}, Q_{3}$ if and only if

$$
\left[Q_{1}, Q_{2}, Q_{3}\right]= \pm 1
$$

Remark. The meaning of $\left[Q_{1}, Q_{2}, Q_{3}\right]=-1$ is clear; it means that the pairs $\left\{q_{j}, q_{k}\right\},\left\{p_{i}, \bar{p}_{i}\right\}$ are harmonic. On the other hand $\left[Q_{1}, Q_{2}, Q_{3}\right]=1$ implies that either $q_{j}$ coincides with $q_{k}$ or $p_{i}$ with $\bar{p}_{i}$; but this is just the case if the common tangents $l_{1}, l_{2}, l_{3}$ meet at one point or if the contact points $p_{1}, p_{2}, p_{3}$ are collinear (on one line). We should remark that both cases can happen simultaneously, and here is a supplement to Proposition 7.1:

Proposition 7.2. Let $Q_{1}, Q_{2}, Q_{3}$ be as in Proposition 7.1. Let $l_{1}, l_{2}, l_{3}$ (resp. $p_{1}, p_{2}, p_{3}$ ) be the common tangents (resp. the contact points) of pairs $\left\{Q_{2}, Q_{3}\right\},\left\{Q_{1}, Q_{3}\right\},\left\{Q_{1}, Q_{2}\right\}$. Then the following statements are equivalent:

(i) There is more than one conic which is in $(2,2)$-contact with $Q_{1}, Q_{2}, Q_{3}$.

(ii) $l_{1}, l_{2}, l_{3}$ meet at one point and $p_{1}, p_{2}, p_{3}$ are collinear.

(iii) There are exactly two conics which are in $(2,2)$-contact with $Q_{1}, Q_{2}, Q_{3}$.

Assume that this is the case and let $Q_{a}, Q_{b}$ be the conics which are in $(2,2)-$ contact with $Q_{1}, Q_{2}, Q_{3}$. By interchanging $Q_{a}$ and $Q_{b}$ if necessary, we have the relations:

$$
\begin{aligned}
& {\left[Q_{3} / Q_{2}\right] \cdot\left[Q_{2} / Q_{1}\right] \cdot\left[Q_{1} / Q_{3}\right]=-\left[Q_{1}, Q_{2}, Q_{3}\right]=-1} \\
& \left(1-\left[Q_{j} / Q_{i}\right]\right) \cdot\left(1-\left[Q_{k} / Q_{i}\right]\right)=1-\left[Q_{a} / Q_{i}\right] \\
& \left(1-\left[Q_{i} / Q_{j}\right]\right) \cdot\left(1-\left[Q_{i} / Q_{k}\right]\right)=1-\left[Q_{i} / Q_{b}\right]
\end{aligned}
$$

where $(i, j, k)$ can be any permutation of $(1,2,3)$. Furthermore $Q_{a}, Q_{b}$ are in $a$ special position if and only if $\left[Q_{a} / Q_{i}\right] \cdot\left[Q_{i} / Q_{b}\right]$ (which does not depend on $i$ ) is equal to 9 . If this is the case, then $\left[Q_{a} / Q_{b}\right]=-3$.

Remark. $Q_{a}$ and $Q_{b}$ can even be in $(2,2)$-contact: they are so if and only if $\left[Q_{i} / Q_{j}\right]=-1$ for $1 \leqq i \neq j \leqq 3$. This last configuration of five conics is projectively unique and gives the maximal number $(=17)$ of tacnodes in the case of five conics.

Remark. Suppose $Q, Q_{1}, Q_{2}, Q_{3}$ are such conics as in Proposition 7.1. Consider the double covering $\pi: \tilde{\boldsymbol{P}}_{2} \rightarrow \boldsymbol{P}_{2}$ branched over $Q_{1} \cup Q_{2} \cup Q_{3} . \quad \widetilde{\boldsymbol{P}}_{2}$ has 
in general three singular points of type $A_{3}$ and six ordinary double points which come from the singularity of the branch locus. The inverse image $\pi^{-1}(Q)$ decomposes into two non-singular rational curves $Q^{\prime}, Q^{\prime \prime}$ each having self-intersection number -2 . Denote by $\tilde{L}$ the total transform of the general line under $\pi$. We see that $\left(\tilde{L}+Q^{\prime}\right)^{2}=4$ and the line bundle associated with this cycle gives a birational morphism of $\tilde{P}_{2}$ into $\boldsymbol{P}_{3}$; this morphism contracts only $Q^{\prime}$ onto an ordinary double point of the image, which is now a quartic surface with three $A_{3}$ 's and seven $A_{1}$ 's.

8. In this section we will study another important type of configurations of four conics; we will observe a curious multiplicative property of our invariants. Suppose we are given four conics $Q_{1}, Q_{2}, Q_{3}, Q_{4}$ such that $Q_{i}$ and $Q_{j}$ are in a special position (resp. in $(2,2)$-contact) if $\{i, j\}=\{1,2\}$ or $\{3,4\}$ (resp. $i \in\{1,2\}$ and $j \in\{3,4\})$. By Proposition 5.2 we have the identities $\left[Q_{2} / Q_{1}\right]^{2}=\left[Q_{1} / Q_{j}\right]$ $\times\left[Q_{j} / Q_{2}\right] \quad(j=3,4), \quad\left[Q_{4} / Q_{3}\right]^{2}=\left[Q_{3} / Q_{i}\right] \cdot\left[Q_{i} / Q_{4}\right] \quad(i=1,2)$. Motivated by this, we set:

$$
\begin{array}{ll}
\left\langle Q_{i}\right\rangle:=\left[Q_{i} / Q_{3}\right] \cdot\left[Q_{4} / Q_{3}\right]\left(=\left[Q_{i} / Q_{4}\right] \cdot\left[Q_{3} / Q_{4}\right]\right), & i=1,2 \\
\left\langle Q_{j}\right\rangle:=\left[Q_{j} / Q_{1}\right] \cdot\left[Q_{2} / Q_{1}\right]\left(=\left[Q_{j} / Q_{2}\right] \cdot\left[Q_{1} / Q_{2}\right]\right), & j=3,4
\end{array}
$$

Then we also have $\left\langle Q_{1}\right\rangle=\left[Q_{2} / Q_{1}\right]^{2} \cdot\left\langle Q_{2}\right\rangle,\left\langle Q_{3}\right\rangle=\left[Q_{4} / Q_{3}\right]^{2} \cdot\left\langle Q_{4}\right\rangle$ and we set

$$
\begin{aligned}
& \left\langle Q_{1}, Q_{2}\right\rangle:=\left\langle Q_{1}\right\rangle \cdot\left[Q_{1} / Q_{2}\right]\left(=\left\langle Q_{2}\right\rangle \cdot\left[Q_{2} / Q_{1}\right]\right) \\
& \left\langle Q_{3}, Q_{4}\right\rangle:=\left\langle Q_{3}\right\rangle \cdot\left[Q_{3} / Q_{4}\right]\left(=\left\langle Q_{4}\right\rangle \cdot\left[Q_{4} / Q_{3}\right]\right) .
\end{aligned}
$$

Proposition 8.1. Under the assumptions and the notation above, we have

$$
\begin{aligned}
& 4 \cdot\left(1+\left\langle Q_{1}, Q_{2}\right\rangle\right) \cdot\left(1+\left\langle Q_{3}, Q_{4}\right\rangle\right) \\
& =\left(1+\left[Q_{1} / Q_{2}\right]\right) \cdot\left(1+\left[Q_{2} / Q_{1}\right]\right) \cdot\left(1+\left[Q_{3} / Q_{4}\right]\right) \cdot\left(1+\left[Q_{4} / Q_{3}\right]\right) \text {. }
\end{aligned}
$$

9. Now, given a configuration of conics, we want to associate with it a graph expressing to some extent the intersection behavior of the conics. The vertices of the graph should represent the conics themselves. Two conics are joined by a double segment in the graph if they intersect in four points; they are joined by a single segment if they are in (2)-contact (i.e. they touch each other just in one point); they are not connected if they are in $(2,2)$-contact. The more special the position of two conics is, the thiner is their connection in the graph. One should note that the graph does not give any information about the occurrence of triple intersection points which are prohibited by the as- 
sumptions of Section 2. Whenever one finds some interesting graph of conics, one should always check whether this last assumption is fulfilled or not, which might sometimes be painful. Here are some examples which illustrate how to associate our graph: For three conics $Q, Q_{1}, Q_{2}$ in Proposition 5.2 we have the graph:

$$
Q \cdot \quad \stackrel{Q_{1}}{\stackrel{0}{Q_{2}}}
$$

in the general case; in the case of (5.4) it degenerates to the following:<smiles>O[Hg]</smiles>

Proposition 6.2 deals with the graphs:

(II)<smiles>O=C1C2CC12</smiles>

( II' $)$<smiles>O[C@H]1O[C@@H]1O</smiles>

The last graph is discussed also in Proposition 7.1. In Proposition 7.2 the following graphs are discussed:

(III)<smiles>[Os]</smiles><smiles>O=C1O[C@@H]1O</smiles>

(III')<smiles>[18O]=[18O]</smiles>

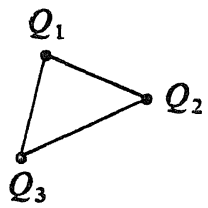

and in the remark following the proposition, the graph:<smiles>[O-][C@@H]1O[C@H]1O</smiles>

For $Q_{1}, Q_{2}, Q_{3}, Q_{4}$ in Section 8.1 we have the graph:

(IV)

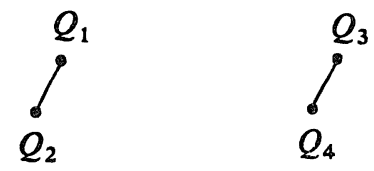


For a given graph we are now interested in the number of parameters on which the corresponding configuration depends. This number is equal to 2, 0, 1, 2, 3, 2, 3 for graphs (IV), (III'), (III'), (III), (II'), (I'), (I) respectively. In all these cases we have enough invariants $\left[Q_{i} / Q_{j}\right],\left[Q_{i}, Q_{j}, Q_{k}\right]$, etc. to describe the moduli space of configurations. In particular there are three parameters for the singular quartic surfaces described in the second remark following Proposition 7.2. This is just in accordance with the fact that, for any moduli space of $K 3$ surfaces, the number of parameters plus the sum of ranks of rational double points on the generic member is always 19 . We should remark here that there are three essentially different types of configurations for the graph (II') i.e. the parameter space has three irreducible components (Proposition 7.1 and the remark following it). One component is self-dual and the other two are dual to each other in the sense of the next section.

10. Let $V$ be a 3-dimensional vector space and $V^{*}$ the dual space of $V$; let $P, P^{*}$ be the projective planes associated with $V, V^{*}$ and $C, C^{*}$ the space of conics in $P, P^{*}\left(C \simeq C^{*} \simeq \boldsymbol{P}_{5}\right)$. Given a non-degenerate quadratic form $q$, we define its dual $q^{*}$ in the following way: Define first the isomorphism $V \ni v$ $\rightarrow \gamma_{q}(v) \in V^{*}$ by setting

$$
\left\langle u, \gamma_{q}(v)\right\rangle=q(u, v)(=\{q(u+v)-q(u)-q(v)\} / 2)
$$

and then set:

$$
q^{*}\left(v^{*}\right)=(\operatorname{det}(q))^{2} \cdot q\left(\gamma_{q}^{-1}\left(v^{*}\right)\right) .
$$

Obviously this induces a birational correspondence

$$
C \ni Q \longleftrightarrow Q^{*} \in C^{*} \quad\left(\left(Q^{*}\right)^{*}=Q\right) .
$$

Since this can be given explicitly by forming the cofactor matrix, we can define $q^{*}$ even in the case when $q$ is of rank $2 ; q^{*}$ is then of rank 1 . This fact will be of some use later. If two conics are in (2)-contact (resp. $(2,2)$-contact), their duals are also in (2)-contact (resp. $(2,2)$-contact). In other words, the graph is invariant under passing to the dual. But lines in $C$ (resp. $C^{*}$ ) are not transformed to lines but to a certain kind of quadratic curves in $C^{*}$ (resp. C). For this reason it is interesting to see how our invariants behave under duality: We begin with the natural one to one correspondence between the references of $Q_{1}, Q_{2} \in C$ and those of $Q_{1}^{*}, Q_{2}^{*} \in C^{*}$. We have assumed that $Q_{1}, Q_{2}$ are in general position. Let $L$ resp. $L^{*}$ be the linear systems generated by $Q_{1}, Q_{2}$ resp. $Q_{1}^{*}, Q_{2}^{*}$. Denote by $K$ 
the image of $L^{*}$ under duality. Since $K$ is a quadratic curve in $C, K$ is contained in a (unique) plane, which we denote by $H . L$ is contained in $H$ as $Q_{1}, Q_{2}$ are on $K$. Now recall that the references $c_{1}, c_{2}, c_{3}$ (resp. $\tilde{c}_{1}, \tilde{c}_{2}, \tilde{c}_{3}$ ) of $\left\{Q_{1}, Q_{2}\right\}$ (resp. $\left\{Q_{1}^{*}, Q_{2}^{*}\right\}$ ) are singular conics in $L$ (resp. in $L^{*}$ ). We denote the images (under duality) of $\tilde{\iota}_{1}, \tilde{c}_{2}, \tilde{\iota}_{3}$ by $\bar{c}_{1}, \bar{\iota}_{2}, \bar{\iota}_{3}$; they lie on $K$ and they are of rank 1 . The last fact implies that the triangle $\Delta$ defined by $\bar{c}_{1}, \bar{c}_{2}, \bar{c}_{3}$ coincides with the set of singular conics in $H$ (The singular conics form a cubic hypersurface of $C \simeq \boldsymbol{P}_{5}$ ). It follows that $\left\{c_{1}, c_{2}, c_{3}\right\}=L \cap \Delta$. By renumbering if necessary, we can assume that $\iota_{i}, \bar{\iota}_{j}, \bar{\iota}_{k}$ are collinear for any permutation $(i, j, k)$ of $(1,2,3)$, and in that case we say that $c_{1}, c_{2}, c_{3}$ correspond to $\tilde{c}_{1}, \tilde{c}_{2}, \tilde{c}_{3}$.

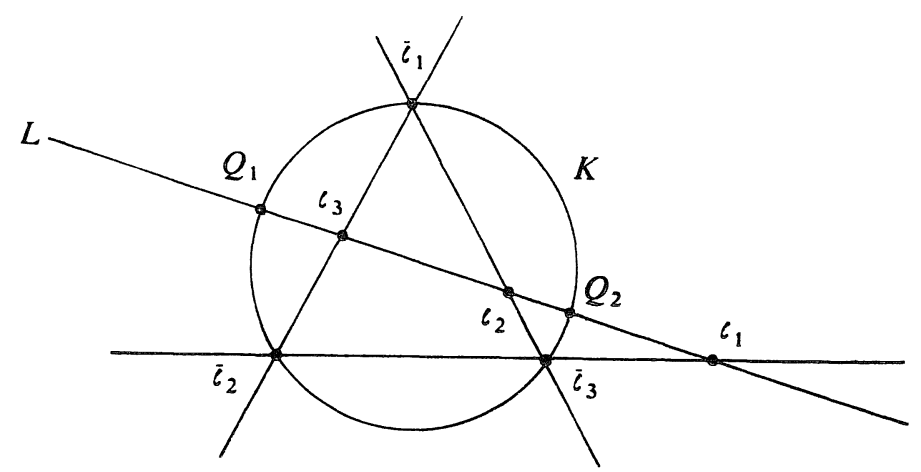

Note that $K$ is isomorphic to $L^{*}$ under duality and that $Q_{1}, Q_{2}, \bar{\iota}_{1}, \bar{c}_{2}, \bar{\iota}_{3}$ correspond to $Q_{1}^{*}, Q_{2}^{*}, \tilde{\iota}_{1}, \tilde{\iota}_{2}, \tilde{\iota}_{3}$ under this isomorphism. Since $L$ and $K$ are also isomorphic by the projection through $\bar{\iota}_{i}$ for each $i$, we can prove:

Proposition 10.1. With the above notation we have

$$
\left[Q_{2} / Q_{1} ; \iota_{i}\right]=\left[Q_{1}^{*} / Q_{2}^{*} ; \tilde{\iota}_{i}\right] .
$$

If $Q_{1}$ and $Q_{2}$ are in a special position, then

$$
\left[Q_{2} / Q_{1}\right]=\left[Q_{1}^{*} / Q_{2}^{*}\right]
$$

11. In Section 6 we have introduced the invariant $\left[Q_{1}, Q_{2}, Q_{3}\right]$ for any configuration of the type:

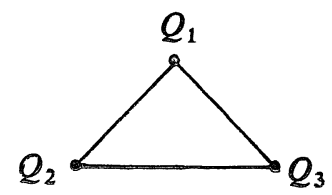


The question presents itself: how can it be well defined in the case of the degenerate graph:

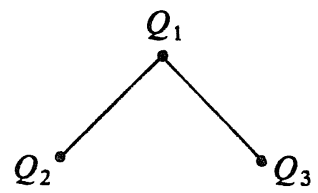

(It should be well defined in view of the identity (7.2) whose left hand side has meaning even in the degenerate case.) Suppose that $Q_{1}, Q_{2}, Q_{3}$ intersect each other in the manner shown by the graph above. The pair $\left\{Q_{1}, Q_{2}\right\}$ (resp. $\left\{Q_{1}\right.$, $\left.Q_{3}\right\}$ ) has only one common tangent $l_{3}$ (resp. $l_{2}$ ) and one contact point $p_{3}$ (resp. $p_{2}$ ) while $\left\{Q_{2}, Q_{3}\right\}$ has two common tangents $l_{1}, l_{1}^{\prime}$ and two contact points $p_{1}$ $\left(\in l_{1}\right), p_{1}^{\prime}\left(\in l_{1}^{\prime}\right)$. We have thus two systems of data $\left\{p_{1}, p_{2}, p_{3} ; l_{1}, l_{2}, l_{3}\right\},\left\{p_{1}^{\prime}, p_{2}\right.$, $\left.p_{3} ; l_{1}^{\prime}, l_{2}, l_{3}\right\}$ to define the invariant $\left[Q_{1}, Q_{2}, Q_{3}\right]$ (Section 6). As is suggested above, we might expect the two quantities obtained from these to coincide, which is actually the case. In particular, in the special case when $\left[Q_{1}, Q_{2}, Q_{3}\right]$ $=1$, we have:

Proposition 11.1. If $p_{1}, p_{2}, p_{3}$ lie on one line (resp. $l_{1}, l_{2}, l_{3}$ meet at one point), then $l_{1}^{\prime}, l_{2}, l_{3}$ meet at one point (resp. $p_{1}^{\prime}, p_{2}, p_{3}$ lie on one line).

Recall that, if $p_{1}, p_{2}, p_{3}$ are collinear, we have one conic which is in $(2,2)$ contact with $Q_{1}, Q_{2}, Q_{3}$, according to Proposition 7.1. Since in that case $l_{1}^{\prime}, l_{2}$, $I_{3}$ also meet in one point, we should have another conic which is also in $(2,2)$ contact with $Q_{1}, Q_{2}, Q_{3}$. But these two coincs must coincide:

Proposition 11.2. There is exactly one (irreducible) family of configurations belonging to the graph:

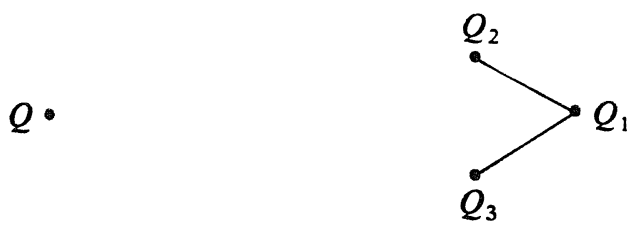

This family has two parameters and is self-dual in the sense of Section 10. We always have $\left[Q_{1}, Q_{2}, Q_{3}\right]=1$.

12. We close this note by discussing some important types of configurations of five conics: Suppose first that conics $Q_{1}, Q_{2}, Q_{3}, Q_{4}, Q_{5}$ intersect as follows: 


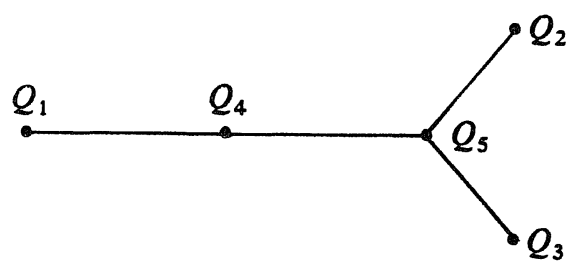

This configuration depends only on one parameter; more precisely, the invariants $\left[Q_{i} / Q_{j}\right],\left[Q_{i}, Q_{j}, Q_{k}\right]$ generate over $Q$ the one-dimensional rational function field over the number field $Q(\sqrt{2})$. One should remark first:

$$
\left[Q_{1}, Q_{4}, Q_{5}\right]=3 \pm 2 \sqrt{2}=(1 \pm \sqrt{2})^{2}
$$

which is obviously a unit. If we set:

$$
a=\frac{1-\left[Q_{1}, Q_{4}, Q_{5}\right] \cdot\left[Q_{5} / Q_{3}\right]}{1-\left[Q_{1}, Q_{5}, Q_{4}\right] \cdot\left[Q_{5} / Q_{2}\right]}
$$

then we have

$$
\left[Q_{5} / Q_{3}\right]=-\left[Q_{1}, Q_{5}, Q_{4}\right] \cdot \frac{a \cdot(a+1)}{a-1}
$$

The other invariants are easily expressed as rational functions of $a$ over $Q(\sqrt{2})$ by Propositions 5.2, 6.2.

Another remarkable fact concerning five conics is the non-existence of the following graph:

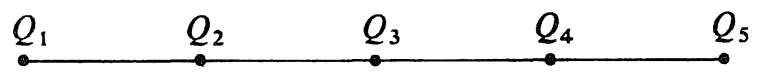

One can actually construct several one-parameter families of configurations belonging to this graph; but some three among $Q_{1}, Q_{2}, \ldots, Q_{5}$ must meet (in fact, touch) at one point, which was excluded by the assumptions of Section 2.

By an elementary argument we can also prove non-existence of the graph
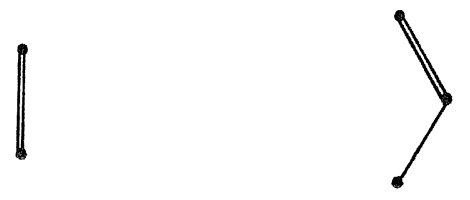

and any of its degenerations.

Finally, we want to mention an example of a graph for which the parameter space of the corresponding configurations is not so easily described; namely we consider the graph: 


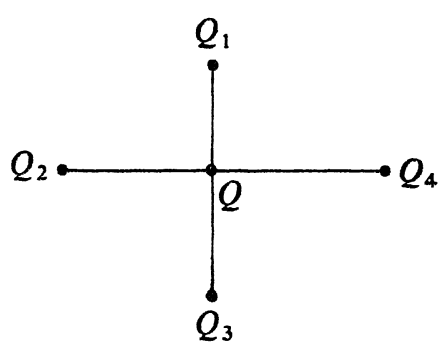

As is remarked in Section 6, the configuration formed by $Q_{1}, Q_{2}, Q_{3}, Q_{4}$ is unique; so we fix these four conics. Now we denote by 2 the totality of conics $Q$ which intersect $Q_{1}, Q_{2}, Q_{3}, Q_{4}$ just as the graph indicates above, and for which the graph becomes admissible with respect to the assumptions of Section 2. For $i=1,2,3$ and $Q \in \mathscr{Q}$ we set:

$$
\tau_{i}(Q)=\left[Q, Q_{i}, Q_{4}\right]
$$

and consider the map

$$
\mathscr{2} \ni Q \longrightarrow \tau(Q):=\left(\tau_{1}(Q), \tau_{2}(Q), \tau_{3}(Q)\right) \in C^{3} \subseteq P_{3}(C) .
$$

The closure in $P_{3}(C)$ of the image of this map is now an irreducible curve of degree 42 and genus 17 . The group $G$ of projective transformations which map each $Q_{i}$ onto itself is of order 4; it acts naturally on $2.2 / G$ is embedded into the curve by $\tau$. Because of this high genus we could hardly perform further experiments concerning this type of configurations. 
